
Sanjeevi

\title{
A Study of Within-Host Dynamics of Dengue Infection incorporating both Humoral and Cellular Response with a Time Delay for Production of Antibodies
}

https://doi.org/10.1515/cmb-2020-0118

Received January 24, 2021; accepted May 17, 2021

Abstract: a. Background: Dengue is an acute illness caused by a virus. The complex behaviour of the virus in human body can be captured using mathematical models. These models helps us to enhance our understanding on the dynamics of the virus.

b. Objectives: We propose to study the dynamics of within-host epidemic model of dengue infection which incorporates both innate immune response and adaptive immune response (Cellular and Humoral). The proposed model also incorporates the time delay for production of antibodies from $\mathrm{B}$ cells. We propose to understand the dynamics of the this model using the dynamical systems approach by performing the stability and sensitivity analysis.

c. Methods used: The basic reproduction number $\left(\mathrm{R}_{0}\right)$ has been computed using the next generation matrix method. The standard stability analysis and sensitivity analysis were performed on the proposed model.

d. Results: The critical level of the antibody recruitment rate $(q)$ was found to be responsible for the existence and stability of various steady states. The stability of endemic state was found to be dependent on time delay $(\tau)$. The sensitivity analysis identified the production rate of antibodies $(q)$ to be highly sensitive parameter.

e. Conclusions: The existence and stability conditions for the equilibrium points have been obtained. The threshold value of time delay $\left(\tau_{0}\right)$ has been computed which is critical for change in stability of the endemic state. Sensitivity analysis was performed to identify the crucial and sensitive parameters of the model.

Keywords: Dengue infection; Cellular and Humoral response; Within-host dynamics; Time delay

MSC: 37N25; 92B05; $92 \mathrm{C} 99$

\section{Introduction}

Dengue is a mosquito-borne viral disease transmitted by female mosquitoes mainly of the species Aedes aegypti and, to a lesser extent, Ae. albopictus. It is an acute illness of sudden onset that usually follows a benign course with symptoms such as headache, fever, exhaustion, severe muscle and joint pain, swollen lymph nodes (lymphadenopathy), and rash. Dengue virus has four serotypes denoted by DEN I, DEN II,

Deva Siva Sai Murari Kanumoori: University of L’Aquila, L’Aquila, Italy

D Bhanu Prakash: Department of Mathematics and Computer Science, Sri Sathya Sai Institute of Higher Learning - SSSIHL, India

${ }^{\star}$ Corresponding Author: D. K. K. Vamsi: Department of Mathematics and Computer Science, Sri Sathya Sai Institute of Higher Learning - SSSIHL, India, E-mail: dkkvamsi@sssihl.edu.in

Carani B Sanjeevi: Vice Chancellor, Sri Sathya Sai Institute of Higher Learning -SSSIHL, India

Department of Medicine, Karolinska Institute, Stockholm, Sweden, E-mail: sanjeevi.carani@sssihl.edu.in, sanjeevi.carani@ki.se

Oopen Access. (ब) BY 02021 Deva Siva Sai Murari Kanumoori et al., published by De Gruyter. This work is licensed under the Creative Commons Attribution alone 4.0 License. 
DEN III and DEN IV. When a person is infected first time by a dengue virus, the immune system against the dengue virus is activated. The infection of dengue virus for the second time by a different serotype leads to secondary infection.

Mathematical models play a crucial role in understanding and controlling the dengue virus. Many vector-host models are developed to study the dengue virus at population level $[2,4,9]$. There are very few works performed to understand the dengue viral dynamics in human body [10, 15]. But, not one of the current models $[6,8,12,13]$ have considered the role of innate immune response in the defence to the dengue viral attack until recently [5] introduced innate immune response and show that it can reproduce the characteristic features of the primary infection.

Innate immune response is the first line of defense mechanism in the human body to fight against any invading pathogen. This is non-specific to invading pathogen. The innate immune system thus produces a type I interferon(IFN), with main interferon's being IFN $\alpha / \beta$. The IFN produced induce resistance in infecting the neighboring uninfected cells thus, limiting the quick spread of the virus $[11,14,16]$. Not only this, the IFN has ability to active the natural killer cells(NK) during the primal stages of the infection, that have ability to destroy infected cells $[14,3,17]$. In dengue, we can detect IFN generally in 24-48 hours after the infection and correlating with the virus titer peak [1]. Thus, IFN produced can act as early indicator for the infection. Dengue patients have also shown early activation of the NK cells $[5,7]$.

In the second stage of infection, the viral pathogen will be carried to the thymus to activate the adaptive immune response which is more powerful and more specific to the invading pathogen. The adaptive immune system takes longer to respond but it provides a long term immunity against the virus $[8,12]$. The adaptive immune system consists of both cellular and humoral responses. In Cellular response, T-cells will clear the virus and in humoral response, B-cells will mature into plasma cells and secrete antibodies that neutralises the antigen-presenting viruses.

In this paper, a non-linear model is proposed which incorporates innate and adaptive (both cellular and humoral) immune responses. The equilibrium points and the stability analysis is carried out. The time delay has been considered during the process of antibody secretion and its further impact on stability of steady states have been discussed. The paper is organized as follows: In section 2, the mathematical model has been formulated. In section 3, model analysis has been performed. In section 4, the sensitivity analysis has been performed. In section 5 , discussion has been given.

\section{Mathematical Model}

The present model is an extension of the model in [10]. Let S, I and V be the number of healthy target cells(monocytes, macrophages or dendritic cells etc), infected cells and dengue virions respectively. To model the innate immune response on dengue viral dynamics, let F denote the interferons (IFN) released by the infected cells. Let $Z$ be the number density of $\mathrm{T}$ immune cells due to adaptive immune response (cellular immune response). The $\mathrm{T}$ cells activate $\mathrm{B}$ cells (humoral immune response) that again differentiate to plasma cells to produce antibodies. Let $B$ and $A$ be the density of $B$ cells and neutralizing antibodies respectively. Let $\tau$ be the time delay in the production of antibodies from B cells. The antibodies bind the virions and neutralize them. 
The dynamics of S, I, V, Z, B, A, F are described in the following system of differential equations.

$$
\begin{aligned}
& \frac{\mathrm{d} S}{\mathrm{~d} t}=\mu-d S-\beta_{1} S V \\
& \frac{\mathrm{d} I}{\mathrm{~d} t}=\beta_{1} S V-d_{1} I-\phi I F \\
& \frac{\mathrm{d} V}{\mathrm{~d} t}=k I-d_{2} V-p_{1} A V \\
& \frac{\mathrm{d} Z}{\mathrm{~d} t}=\beta_{2} Z I-d_{3} Z \\
& \frac{\mathrm{d} B}{\mathrm{~d} t}=\eta+c_{1} B Z-d_{4} B \\
& \frac{\mathrm{d} A}{\mathrm{~d} t}=q B(t-\tau)-d_{5} A \\
& \frac{\mathrm{d} F}{\mathrm{~d} t}=q_{1} I-d_{6} F
\end{aligned}
$$

The values for parameters are given in table 1. Using the parameter values in table 1 , the dynamics of all the compartments (S, I, V, Z, B, A, F) from the initial point $(S, I, V, Z, B, A, F)=(200,50,100,6,50,0,0)$ are given in the figure 1.

Table 1: Table describing parameter values

\begin{tabular}{c|c|c|c}
\hline Variable & Description & Value & Source \\
\hline$\mu$ & Rate of production of susceptible cells & 10 & {$[10]$} \\
\hline $\mathrm{d}$ & Death rate of target cells & 0.05 & {$[10]$} \\
\hline $\mathrm{d}_{1}$ & Death rate of infected cells & 0.5 & {$[10]$} \\
\hline $\mathrm{d}_{2}$ & Death rate of virus particles & 0.5 & {$[10]$} \\
\hline $\mathrm{d}_{3}$ & Death rate of immune cells & 0.002 & {$[10]$} \\
\hline $\mathrm{d}_{4}$ & Death rate of B cells & 0.049 & {$[10]$} \\
\hline $\mathrm{d}_{5}$ & Death rate of antibodies & 0.051 & {$[10]$} \\
\hline $\mathrm{d}_{6}$ & Death rate of IFN & 0.7 & {$[15]$} \\
\hline$\beta_{1}$ & Transformation rate of target cells to infected cells & 0.001 & {$[10]$} \\
\hline$\beta_{2}$ & Activation rate of immune response due to infected cells & 0.005 & {$[10]$} \\
\hline $\mathrm{c}_{1}$ & Rate of activation of B cells by T immune cells & 0.001 & {$[10]$} \\
\hline $\mathrm{p}_{1}$ & Rate at which antibodies neutralise virions & 0.001 & {$[10]$} \\
\hline $\mathrm{q}$ & Rate of production of antibodies by infected cells & 0.3 & {$[10]$} \\
\hline $\mathrm{q}_{1}$ & Rate of production of IFN(F) by infected cells & 0.8 & {$[15]$} \\
\hline$\eta$ & Rate of production of B-cells & 10 & {$[10]$} \\
\hline $\mathrm{k}$ & Burst rate of virus particles & 5 & {$[10]$} \\
\hline$\phi$ & Rate at which infected cells are killed by NK-cells & 0.002 & {$[15]$} \\
\hline & & &
\end{tabular}

\section{Mathematical Analysis}

\section{Positivity of Solutions}

For any mathematical model it is fundamental to show that the system of equations considered are positive and has bounded solutions. We now show that if the initial conditions of the system (1) - (7) are positive, then the solution remain positive for any future time. 

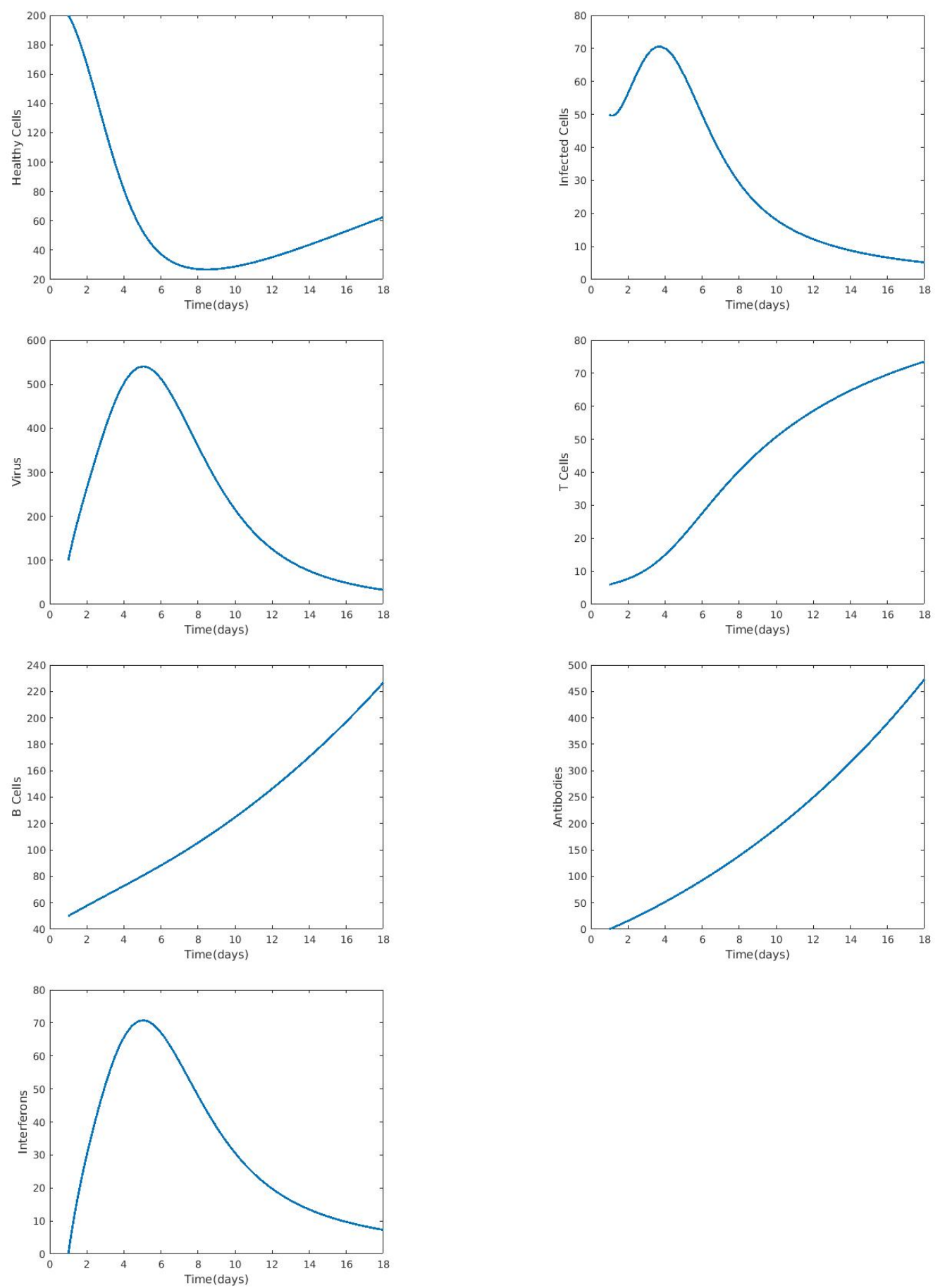

Figure 1: The dynamics of S, I, V, Z, B, A, F. The parameter values are as in table 1. Initial Conditions $(S, I, V, Z, B, A, F)=$ $(200,50,100,6,50,0,0)$.

Using the equations (1) - (7), we get,

$$
\begin{aligned}
& \left.\frac{\mathrm{d} S}{\mathrm{~d} t}\right|_{S=0}=\mu \geq 0 \\
& \left.\frac{\mathrm{d} I}{\mathrm{~d} t}\right|_{I=0}=\beta_{1} S V \geq 0 \\
& \left.\frac{\mathrm{d} V}{\mathrm{~d} t}\right|_{V=0}=k I \geq 0 \\
& \left.\frac{\mathrm{d} Z}{\mathrm{~d} t}\right|_{Z=0} \geq 0 \\
& \left.\frac{\mathrm{d} B}{\mathrm{~d} t}\right|_{B=0}=\eta \geq 0 \\
& \left.\frac{\mathrm{d} A}{\mathrm{~d} t}\right|_{A=0}=q B(t-\tau) \geq 0 \\
& \left.\frac{\mathrm{d} F}{\mathrm{~d} t}\right|_{F=0}=q_{1} I \geq 0
\end{aligned}
$$


Thus all the above rates are non-negative on the bounding planes (given by $S=0, I=0, V=0, Z=0, B=0$, $A=0, F=0$ ) of the non-negative region of the real space. So, if a solution begins in the interior of this region, it will remain inside it throughout time t. This happens because the direction of the vector field is always in the inward direction on the bounding planes as indicated by the above inequalities. Hence, we conclude that all the solutions of the the system (1) - (7) remain positive for any time $t>0$ provided that the initial conditions are positive. Next we will show that the solution is bounded with each of the bounded control variables.

\section{Boundedness of Solutions}

Let $\mathrm{N}(\mathrm{t})=\mathrm{S}(\mathrm{t})+\mathrm{I}(\mathrm{t})+\mathrm{V}(\mathrm{t})+\mathrm{Z}(\mathrm{t})+\mathrm{B}(\mathrm{t})+\mathrm{A}(\mathrm{t})+\mathrm{F}(\mathrm{t})$. Now,

$$
\frac{\mathrm{d} N}{\mathrm{~d} t}=\frac{\mathrm{d} S}{\mathrm{~d} t}+\frac{\mathrm{d} I}{\mathrm{~d} t}+\frac{\mathrm{d} V}{\mathrm{~d} t}+\frac{\mathrm{d} Z}{\mathrm{~d} t}+\frac{\mathrm{d} B}{\mathrm{~d} t}+\frac{\mathrm{d} A}{\mathrm{~d} t}+\frac{\mathrm{d} F}{\mathrm{~d} t} \leq 0
$$

As analytically it is tough to show that $N(t)=C$ for a constant $C$, we show that the system (1) - (7) is a bounded space by simulating the system at different initial points such as $P 1=$ $(160,0.2,0.05,3,150,100,2), P 2=(180,0.3,2,8,200,111,4), P 3=(192,0.1,0.5,6,240,140,6)$. These plots are shown in figure 2.

Therefore the biologically feasible region is given by the following set,

$$
\Sigma=\{(S(t), I(t), V(t), Z(t), B(t), A(t), F(t)): N(t) \leq C, t \geq 0\}
$$

\section{Basic reproductive number}

The basic reproductive number for the model (1) - (7) is defined as the average number of infected target cells generated by a single infected cell placed in an uninfected target cell population [13]. Basic reproductive number computed by next generation approach is obtained as

$$
\mathcal{R}_{0}=\frac{\beta_{1} d_{4} d_{5} \mu k}{d d_{1}\left(d_{2} d_{4} d_{5}+\eta q p_{1}\right)}
$$

\subsection{Equilibrium states and their stability}

Three equilibrium solutions are obtained for the dengue model (1) - (7).

\subsubsection{Infection-free state( $\left.E_{0}\right)$}

The equilibrium densities for the state $\mathrm{E}_{0}$ is given as

$$
E_{0}=\left((\widehat{S}, 0,0,0, \widehat{B}, \widehat{A}, 0) ; \widehat{S}=\frac{\mu}{d}, \widehat{B}=\frac{\eta}{d_{4}}, \widehat{A}=\frac{\eta q}{d_{4} d_{5}}\right.
$$

Now, for the stability analysis, the jacobian matrix $(J)$ for the system (1) - (7) is computed as

$$
J[E]=\left[\begin{array}{ccccccc}
-\left(d+\beta_{1} V\right) & 0 & -\beta_{1} S & 0 & 0 & 0 & 0 \\
-\beta_{1} V & -\left(d_{1}+\phi F\right) & \beta_{1} S & 0 & 0 & 0 & -\phi I \\
0 & k & -\left(d_{2}+p_{1} A\right) & 0 & 0 & -p_{1} V & 0 \\
0 & \beta_{2} Z & 0 & \beta_{2} I-d_{3} & 0 & 0 & 0 \\
0 & 0 & 0 & c_{1} B & c_{1} Z-d_{4} & 0 & 0 \\
0 & 0 & 0 & 0 & q e^{(-\lambda \tau)} & -d_{5} & 0 \\
0 & q_{1} & 0 & 0 & 0 & 0 & -d_{4}
\end{array}\right]
$$



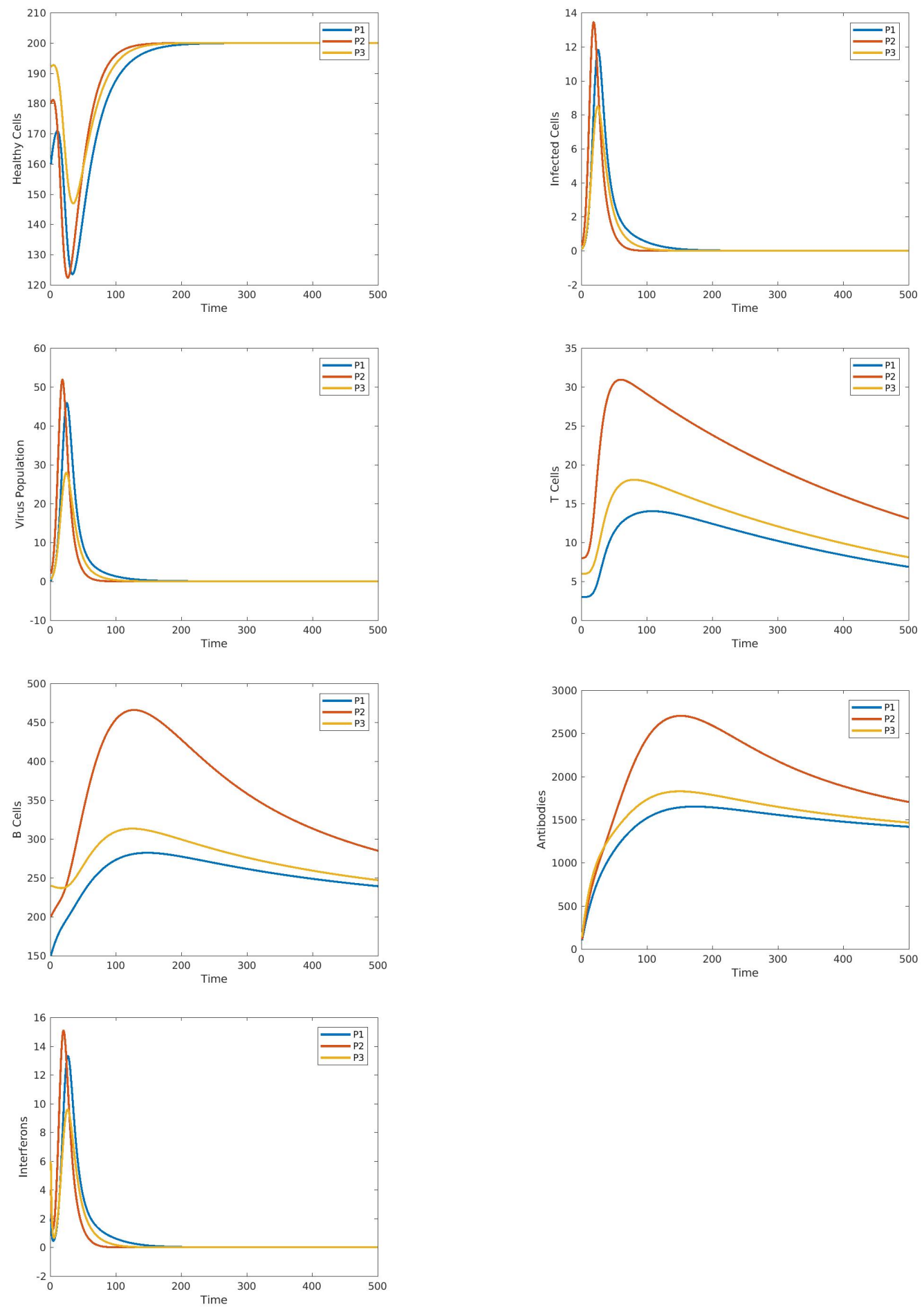

Figure 2: The dynamics of S, I, V, Z, B, A, F at various initial points P1, P2, P3. The parameter values are as in table 1.

The characteristic equation about infection free state is given as

$$
(\lambda+d)\left(\lambda+d_{3}\right)\left(\lambda+d_{4}\right)\left(\lambda+d_{5}\right)\left(\lambda+d_{6}\right) G(\lambda)=0
$$


where,

$$
G(\lambda)=\lambda^{2}+\lambda\left(d_{1}+d_{2}+\frac{p_{1} \eta q}{d_{4} d_{5}}\right)+d_{1} d_{2}\left(1+\frac{p_{1} \eta q}{d_{2} d_{4} d_{5}}\right)\left(1-\mathcal{R}_{0}\right)
$$

From the above equation 10, the following observations are made:

a. It is independent of time delay.

b. When $\mathcal{R}_{0}<1$, all the eigenvalues are negative. Therefore, disease-free state is locally asymptotically stable.

c. When $\mathcal{R}_{0}>1$, the two eigenvalues will have negative real parts provided

Then,

$$
d_{1} d_{2}\left(1+\frac{p_{1} \eta q}{d_{2} d_{4} d_{5}}-\mathcal{R}_{0}\right)>0
$$

$$
q>\frac{\left(\mathcal{R}_{0}-1\right) d_{2} d_{4} d_{5}}{p_{1} \eta}\left(=q_{c}\right)
$$

Thus, even for $\mathcal{R}_{0}>1$, there is the critical level of antibodies $\left(\mathrm{q}_{c}\right)$ over which infection will die out and the $\mathrm{E}_{0}$ becomes locally asymptotically stable. If $\mathrm{q} \leq \mathrm{q}_{c}$, then $\mathrm{E}_{0}$ will be unstable.

\subsubsection{Ineffective cellular immune response state $\left(E_{1}\right)$}

The expression for this state $\mathrm{E}_{1}=(\bar{S}, \bar{I}, \bar{V}, 0, \bar{B}, \bar{A}, \bar{F}$, $)$ are obtained as:

$$
\begin{gathered}
\bar{S}=\frac{\mu}{d+\beta_{1} \bar{V}} ; \bar{V}=\frac{d d_{1} \mathcal{R}_{0}}{\beta_{1} \mu} \bar{I} ; \bar{F}=\frac{q_{1}}{d_{6}} \bar{I} ; \bar{B}=\frac{\eta}{d_{4}} ; \bar{A}=\frac{\eta q}{d_{4} d_{5}} ; \\
\bar{I}=\frac{d_{6}}{2 \phi q_{1} d_{1} \mathcal{R}_{0}}\left[-\left(d_{1}^{2} \mathcal{R}_{0}+\frac{\mu \phi q_{1}}{d_{6}}\right)+\sqrt{\left(d_{1}^{2} \mathcal{R}_{0}+\frac{\mu \phi q_{1}}{d_{6}}\right)^{2}-4 \frac{\phi q_{1} d_{1} \mu d_{1}}{d_{6}} \mathcal{R}_{0}\left(1-\mathcal{R}_{0}\right)}\right]
\end{gathered}
$$

The existence condition for $\mathrm{E}_{1}$ becomes : $\mathcal{R}_{0}>1$. This is because only then we can have $\bar{I} \geq 0$,which is our initial condition.

The characteristic equation about the state $\mathrm{E}_{1}$ is given as:

$$
\left(\lambda+d_{3}-\beta_{2} I\right)\left(\lambda+d_{4}\right)\left(\lambda+d_{5}\right)\left(\lambda^{4}+A_{1} \lambda^{3}+A_{2} \lambda^{2}+A_{3} \lambda+A_{4}\right)=0
$$

where,

$$
\begin{gathered}
A_{1}=\left(d+d_{1}+d_{2}+d_{6}+\frac{p_{1} \eta q}{d_{4} d_{5}}\right)+\left(\frac{d d_{1} \mathcal{R}_{0}}{\mu}+\frac{\phi q_{1}}{d_{6}}\right) I \\
A_{2}=\left[1+d d_{1}+d d_{2}+d d_{6}+d_{1} d_{2}+d_{1} d_{6}+d_{2} d_{6}+\left(d+d_{1}+d_{6}\right) \frac{p_{1} \eta q}{d_{4} d_{5}}\right]+\left[\frac{\phi q_{1} d d_{1} \mathcal{R}_{0}}{\mu d_{6}}\right] I^{2}+ \\
{\left[\left(d+d_{2}+d_{6}\right) \frac{\phi q_{1}}{d_{6}}+\left(d_{1}+d_{2}+d_{6}\right) \frac{d d_{1} \mathcal{R}_{0}}{\mu}+\frac{p_{1} \eta q}{d_{4} d_{5}}\left(\frac{d d_{1} \mathcal{R}_{0}}{\mu}+\frac{\phi q_{1}}{d_{6}}\right)\right] I-\left[\frac{k \mu \beta_{1}}{d+\frac{d d_{1} \mathcal{R}_{0}}{\mu} I}\right]} \\
A_{3}=\left[d\left(1+d_{1} d_{6}\right) \frac{\beta_{1} \mu k}{d d_{1} \mathcal{R}_{0}}\left(1+d d_{1}+d d_{6}+d_{1} d_{6}\right)\right]+\left[d \phi q_{1}+d_{1} \beta k+d_{6} k+\right. \\
\left.\frac{d d_{1} \mathcal{R}_{0}}{\mu}\left(1+d_{1} d_{6}\right)+\frac{\beta_{1} \mu k \phi q_{1}}{d d_{1} d_{6} \mathcal{R}_{0}}\left(d+d_{6}\right)\right] I+\left[1+\frac{d_{2} d_{4} d_{5}+\eta q p_{1}}{d_{4} d_{5} d_{6}}\right] \frac{\phi q_{1} d d_{1} \mathcal{R}_{0}}{\mu} I^{2} \\
-k \beta_{1}\left(d+d_{6}\right)\left(\frac{\mu}{d+\frac{d d_{1} \mathcal{R}_{0} I}{\mu}}\right) \\
A_{4}=\left(\phi q_{1} \beta_{1} k\right) I^{2}+\left(\beta_{1} k+\beta_{1} k d_{1} d_{6}+\frac{\beta_{1} \mu k \phi q_{1}}{d_{1} \mathcal{R}_{0}}\right) I+\frac{\beta_{1} \mu k}{d_{1} \mathcal{R}_{0}}\left(1+d_{1} d_{6}\right)-k \beta_{1} d d_{6} S
\end{gathered}
$$

Now it is observed from the above characteristic equation that all the eigenvalues are found to be negative provided $\mathrm{q}>\mathrm{q}_{c}$. 


\subsubsection{Endemic equilibrium state( $\left(E^{\star}\right)$}

The expression for this state $\mathrm{E}^{\star}=\left(S^{\star}, I^{\star}, V^{\star}, Z^{\star}, B^{\star}, A^{\star}, F^{\star}\right)$ are obtained as:

$$
\begin{gathered}
S^{\star}=\frac{1}{d}\left(\mu-\frac{d_{1} d_{3}}{\beta_{2}}-\frac{\phi q_{1} d_{3}^{2}}{d_{6} \beta_{2}^{2}}\right) ; I^{\star}=\frac{d_{3}}{\beta_{2}} ; V^{\star}=\frac{1}{\beta_{1}}\left(\frac{\mu}{S^{\star}}-d\right) ; \\
Z^{\star}=\frac{1}{c_{1}}\left(d_{4}-\frac{\eta}{B^{\star}}\right) ; B^{\star}=\frac{d_{5}}{q} A^{\star} ; A^{\star}=\frac{1}{p_{1}}\left(\frac{k d_{3}}{\beta_{2} V^{\star}}-d_{2}\right) ; F^{\star}=\frac{q_{1} d_{3}}{\beta_{2} d_{6}}
\end{gathered}
$$

The endemic equilibrium point $\left(E^{\star}\right)$ exists for the following conditions to be satisfied,

$$
k \beta_{1} \beta_{2}^{2} d_{6} \mu>\left(k d_{3} \beta_{1}+d d_{2} \beta_{2}\right)\left(\phi q_{1} d_{3}+\beta_{2} d_{1} d_{6}\right)
$$

and

$$
d_{1} d_{6} \mu \beta_{2}^{2} \mathcal{R}_{0}>\left(\mu \beta_{2}+d_{1} d_{3} \mathcal{R}_{0}\right)\left(\phi q_{1} d_{3}+\beta_{2} d_{1} d_{6}\right)
$$

Further, to analyze the stability of endemic state,the characteristic equation about endemic state is given as:

$$
\lambda^{7}+C_{1} \lambda^{6}+C_{2} \lambda^{5}+C_{3} \lambda^{4}+C_{4} \lambda^{3}+C_{5} \lambda^{2}+C_{6} \lambda+e^{-\lambda \tau}\left(D_{1} \lambda^{2}+D_{2} \lambda+D_{3}\right)=0
$$

where,

$$
\begin{gathered}
C_{1}=B_{1}+\left(\frac{\eta}{B^{\star}}+d_{5}\right) ; \\
C_{2}=B_{2}+\left(\frac{\eta}{B^{\star}}+d_{5}\right) B_{1}+\frac{\eta d_{5}}{B^{\star}} ; \\
C_{3}=B_{3}+\left(\frac{\eta}{B^{\star}}+d_{5}\right) B_{2}+\frac{\eta d_{5}}{B^{\star}} B_{1} ; \\
C_{4}=B_{4}+\left(\frac{\eta}{B^{\star}}+d_{5}\right) B_{3}+\frac{\eta d_{5}}{B^{\star}} B_{2} ; \\
C_{5}=\frac{\eta}{B^{\star}} B_{4}+\left(\frac{\eta}{B^{\star}}+d_{5}\right) B_{4}+\frac{\eta d_{5}}{B^{\star}} B_{3} ; \\
C_{6}=\frac{\eta d_{5}}{B^{\star}} B_{4} ; \\
D_{1}=\Theta ; D_{2}=\Theta\left(d+d_{6}\right) ; D_{3}=d d_{6} \Theta ; \\
\Theta=\frac{k \beta_{1} d_{3} d_{4} d_{5}}{d}\left[\mu-\left(d_{1}+\frac{\phi q_{1} d_{3}}{\beta_{2} d_{6}}\right)\left(\frac{d_{3}}{\beta_{2}}+\frac{\mu}{d d_{1} \mathcal{R}_{0}}\right)\right] \\
B_{1}=d_{1}+d_{6}+\frac{\phi q_{1} d_{3}}{\beta_{2} d_{6}}+\frac{\mu}{S^{\star}}+\frac{k \beta_{1} \beta_{2} d_{6} S^{\star}}{\phi q_{1} d_{3}+\beta_{2} d_{1} d_{6}} \\
B_{2}=1+d_{1} d_{6}+\frac{\phi q_{1} d_{3}}{\beta_{2} d_{6}}\left(d_{6}+\frac{k d_{3}}{\beta_{2} V^{\star}}+\frac{\mu}{S^{\star}}\right)+\frac{k d_{3}}{\beta_{2} V^{\star}}\left(\frac{\mu}{S^{\star}}+d_{1}+d_{6}\right)+\frac{\mu}{S^{\star}}\left(d_{1}+d_{2}+d_{6}\right) \\
B_{3}=\frac{\mu}{S^{\star}}+\frac{k d_{3}}{\beta_{2} V^{\star}}+\frac{k d_{3} d_{6}}{\beta_{2} V^{\star}}\left(d_{1}+\frac{\phi q_{1} d_{3}}{\beta_{2} d_{6}}+\frac{\mu}{S^{\star}}\right)+\left(d_{6}+\frac{k d_{3}}{\beta_{2} V^{\star}}\right)\left(\frac{\mu d}{S^{\star}}+\frac{\mu \phi q_{1} d_{3}}{\beta_{2} d_{6} S^{\star}}\right)-k \beta_{1}\left(d+d_{6}\right) S^{\star} \\
B_{4}=\frac{\mu k d_{3}}{\beta_{2} S^{\star} V^{\star}}+\frac{d_{1}^{2} d_{2} d_{3} d_{6}}{\beta_{2} S^{\star}}+\frac{k d d_{1} d_{3} d_{6}}{\beta_{2} V^{\star}}+\frac{\phi q_{1} d_{2} d_{3}^{2}}{\beta_{2}^{2} S^{\star}}\left(1+d_{1}+\frac{\phi q_{1}}{d_{6}}\right)+\frac{q_{1} k d d_{3}^{2} \phi}{\beta_{2}^{2} V^{\star}}+ \\
\frac{d_{3}}{\beta_{2}^{3} d_{6} S^{\star}}\left(\phi q_{1} d_{3}+\beta_{2} d_{1} d_{6}\right)^{2}\left(\frac{k d_{3}}{\beta_{2} V^{\star}}-d_{2}\right)-k \beta_{1} d d_{6} S^{\star}
\end{gathered}
$$

In the absence of delay,the characteristic equation (12) becomes

$$
\lambda^{7}+C_{1} \lambda^{6}+C_{2} \lambda^{5}+C_{3} \lambda^{4}+C_{4} \lambda^{3}+C_{5} \lambda^{2}+C_{6} \lambda+\left(D_{1} \lambda^{2}+D_{2} \lambda+D_{3}\right)=0
$$

Since the terms are very lengthy and complex, Routh-Hurwitz criteria for determining the sign of $\lambda$ is of little use. Hence, we numerically show the stability of $E^{\star}$ in the absence of delay. This is shown in figure 3 . 


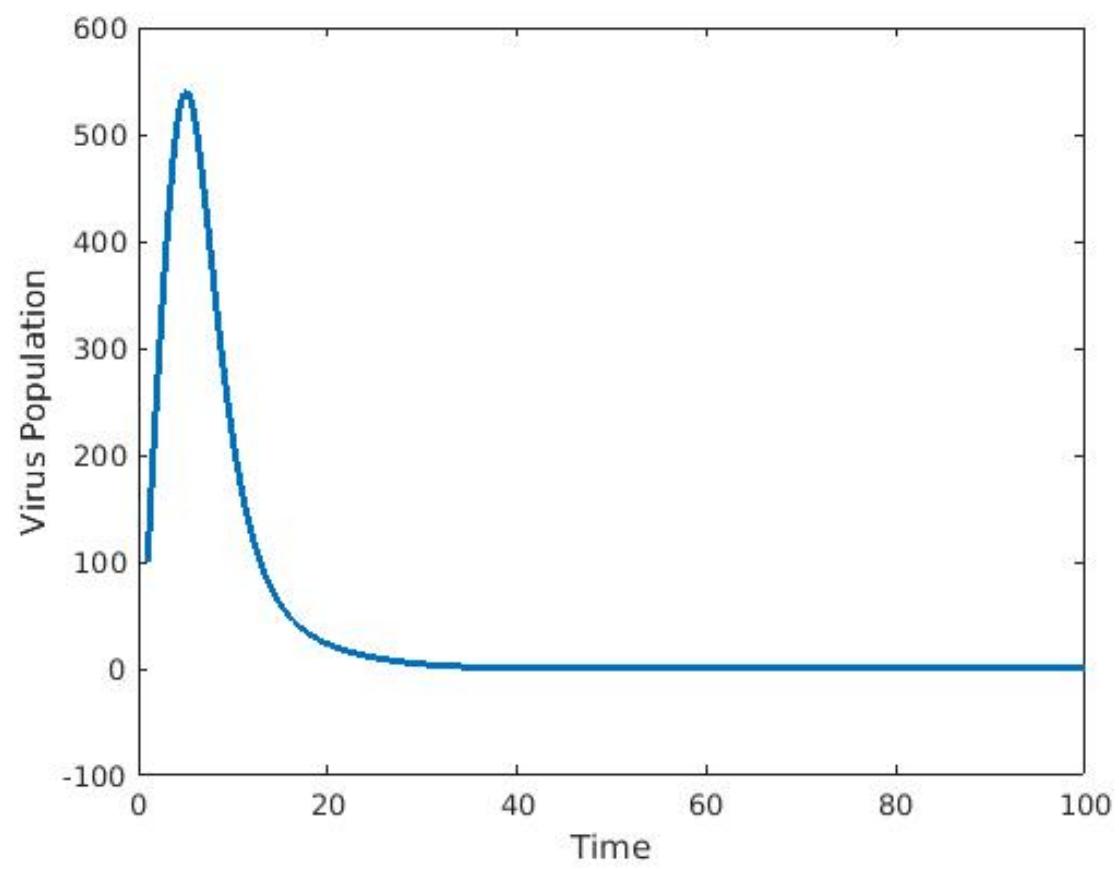

Figure 3: Starting from the initial point $P=(200,50,100,6,50,0,0)$, the time series for virus count showing the stability of $E^{\star}$ when $\tau=0$.

When delay is present, the characteristic equation is modified by substituting $\lambda=i \omega$ as

$$
\omega^{14}+E_{1} \omega^{12}+E_{2} \omega^{10}+E_{3} \omega^{8}+E_{4} \omega^{6}+E_{5} \omega^{4}+E_{6} \omega^{2}+E_{7}=0
$$

where,

$$
\begin{aligned}
& E_{1}=C_{1}^{2}-2 C_{2} ; \\
& E_{2}=C_{2}^{2}-2 C_{1} C_{3}+2 C_{4} ; \\
& E_{3}=C_{3}^{2}-2 C_{4} C_{2}+2 C_{1} C_{5}-2 C_{6} ; \\
& E_{4}=C_{4}^{2}-2 C_{3} C_{5}+2 C_{2} C_{6} ; \\
& E_{5}=C_{5}^{2}-2 C_{2} C_{4}-D_{1}^{2} ; \\
& E_{6}=C_{6}^{2}-D_{2}^{2}+2 D_{1} D_{3} ; \\
& E_{7}=-D_{3}^{2} ;
\end{aligned}
$$

For this equation to have at least one positive root, $E_{7}<0$. Thus, there will exist a positive root $\omega_{0}$, which will satisfy the equation (14). It is given as:

$$
\tau_{j}=\frac{1}{\omega_{0}} \arccos \left[\frac{\Theta_{1}}{D_{1}^{2} \omega^{4}+\left(D_{2}^{2}-2 D_{1} D_{3}\right) \omega^{2}+D_{3}^{2}}\right]+\frac{2 j \pi}{\omega_{0}}, j=0,1,2, \ldots
$$

where,

$$
\Theta_{1}=\left(D_{2}-C_{1} D_{1}\right) \omega^{8}+\left(C_{1} D_{3}-C_{2} D_{2}+C_{3} D_{1}\right) \omega^{6}+\left(-C_{5} D_{1}+C_{4} D_{2}-C_{3} D_{3}\right) \omega^{4}+\left(-C_{6} D_{2}+C_{5} D_{3}\right) \omega^{2}
$$

The corresponding critical delay with the parameters as in table 1 is computed as $\tau_{0}=12.3168$. We now show numerical simulations in figure 4 that the virus count converges to $E^{\star}$ from the initial point $P=(200,50,100,6,50,0,0)$ and exhibits a stable behaviour when $\tau<\tau_{0}$. But, the system admit periodic solution or more complex behavior when $\tau>\tau_{0}$. Thus, the system undergoes Hopf-bifurcation with the critical delay given by equation 15 . 

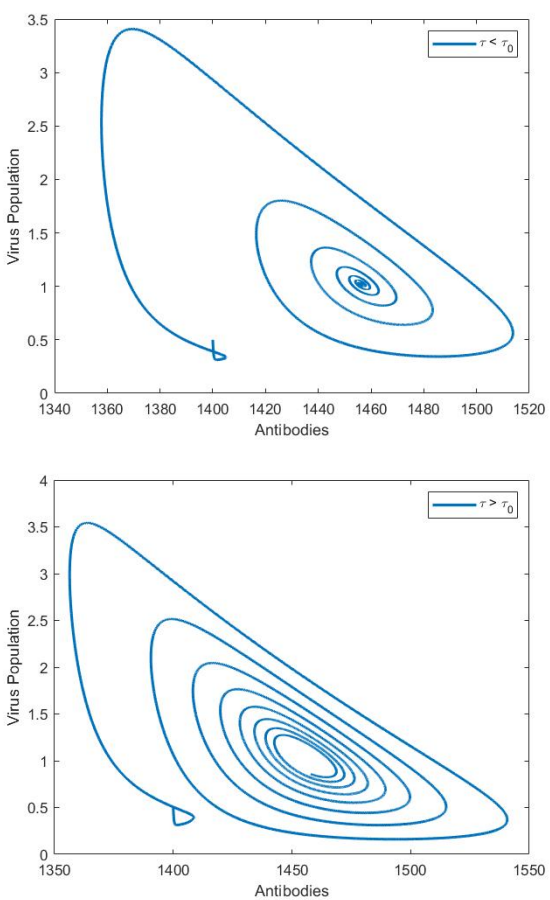
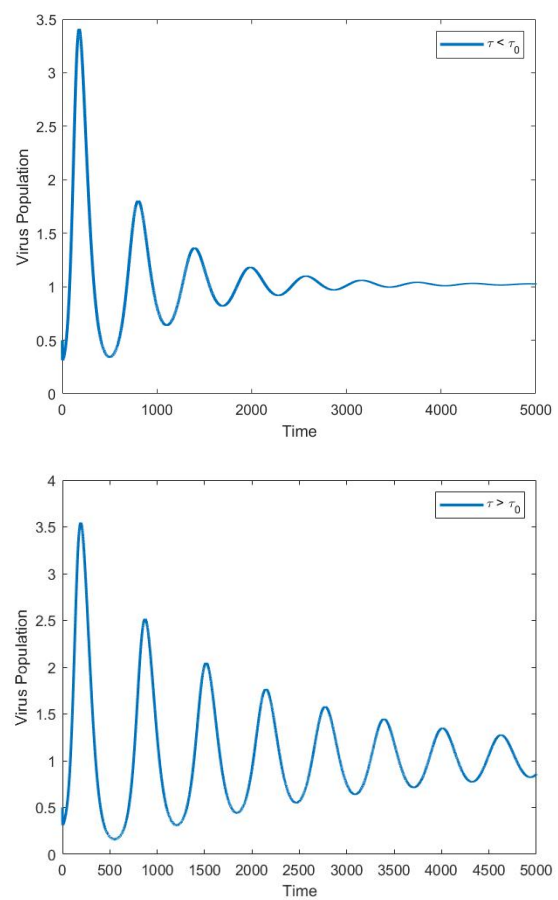

Figure 4: The virus population when $\tau<\tau_{0}$ and $\tau>\tau_{0}$.

\section{Sensitivity Analysis}

\section{Sensitivity Analysis of Rate of Infection $\left(\beta_{1}\right)$}

To analyze the sensitivity of the model with respect to the rate of infection (the rate at which healthy cells are converted to infected cells due to interaction with virus particles $\left(\beta_{1}\right)$, we solve the model numerically for randomized values of $\beta_{1}$. Using the method mentioned in [15], we can analyze how sensitive the system reacts to fluctuations in $\beta_{1}$. Here we use initial conditions (S, I, V, Z, B, A, F) $=(192,0.1,0.5,6,240,1400,6)$ and the parameter values from table 1. Figures 5, 6 contains two subplots each, where the first subplot the time series for virus count at random values of $\beta_{1}$ in the respective ranges. The second subplot gives the mean value of virus count in the range of $\beta_{1}$. From figure 5 , the mean value of virus count reaches peak around five days and declines slowly till 10 days. From figure 6, the mean value of virus count reaches peak value initially and declines very rapidly in the short span of 5-10 days. Further, by comparing figure 5, figure 6 it is observable that, for small values of $\beta_{1}$, the infection takes longer time to clear when compared with the larger values of $\beta_{1}$. Thus the model is stable with respect to the infection rate $\beta_{1}$.

\section{Sensitivity Analysis of Burst Rate of Virus Particles(k)}

Here the variation dynamics of model (1) - (7) with respect to the burst rate of virus particles, $\mathrm{k}$, is considered. The time series for virus count at random values of $k$ and the mean value of the virus count is given in the two subplots in figure 7. This plot means that for various values of $\mathrm{k}$, the mean virus count declines faster before 5 days very rapidly. From this plot, it can be inferred that the burst rate of particles has a predictable effect on the behaviour of the virus count.

\section{Sensitivity Analysis of Production Rate of Antibodies(q)}

Figures 8,9 contains two subplots each, where the first subplot the time series for virus count at random values of $q$ in the respective ranges. The second subplot gives the mean value of virus count in the range of 
q. From figure 8, the mean virus count remains low till 5 days and starts increasing after that. As the value of q increases, from figure 9, the mean virus count starts decreasing from first day and reaches to the minimum by 15 days. Further, by comparing figure 8 , figure 9 it is observable that, for small values of $q$, the mean virus increases over time. As the value of q increases from 0.4, the mean virus count starts decreasing and reaches to the minimum. Thus we can infer that the system is highly sensitive for changes in the parameter q.
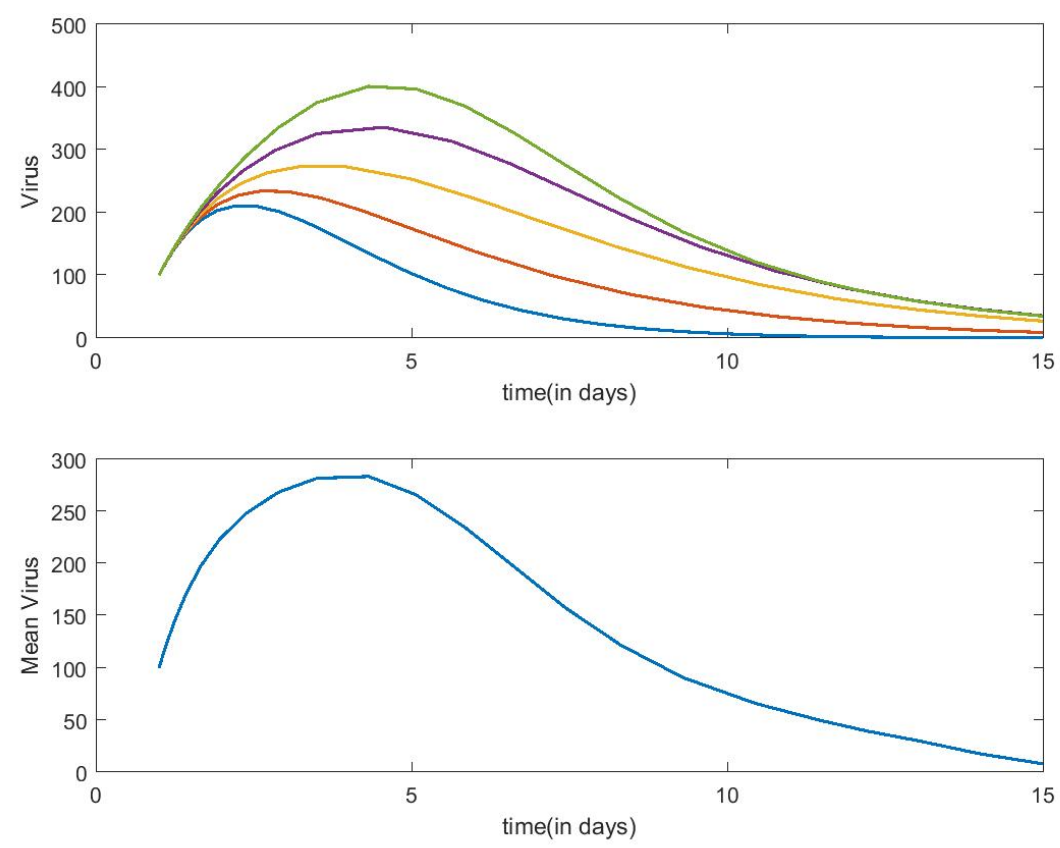

Figure 5: The virus count for values of $\beta_{1}$ from 0 to 0.001 and the mean value of the virus count. The rest of the parameters are chosen from table 1.

\section{Results}

From the above mathematical analysis it can be seen that the critical level of the antibody recruitment rate(q) has been found to be responsible for the existence and stability of various steady states. The stability of endemic state was found to be dependent on time delay $(\tau)$. The critical value of time $\operatorname{delay}\left(\tau_{0}\right)$ was obtained from Hopf bifurcation analysis.

Findings from the sensitivity analysis of several key parameters such as $\beta_{1}, \mathrm{k}, \mathrm{q}$ that are thought to play a significant role in dengue infection are the following:

- The production rate of antibodies (q) was found to be highly sensitive.

- It was observed that the burst rate of virus particles $(\mathrm{k})$ has a predictable behaviour and the rate of infection $\left(\beta_{1}\right)$ is sensitive only in some interval range. 

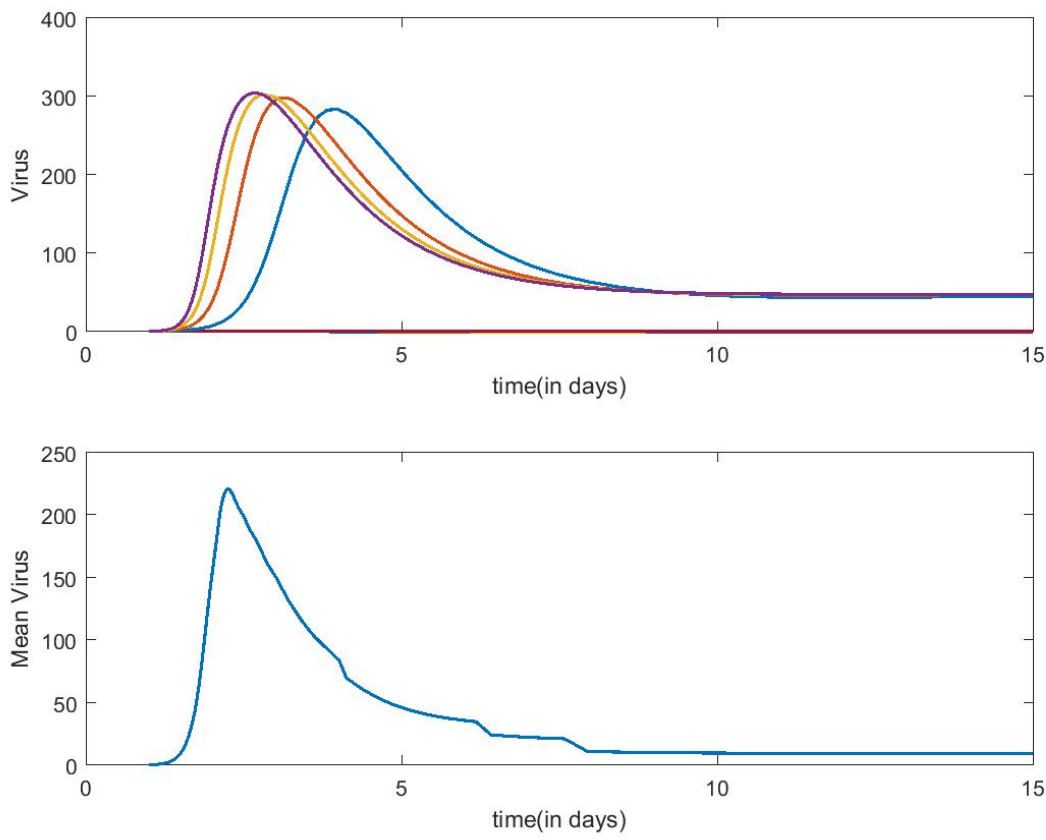

Figure 6: The virus count for values of $\beta_{1}$ from 0.0012 to 0.1 and the mean value of the virus count. The rest of the parameters are chosen from table 1.
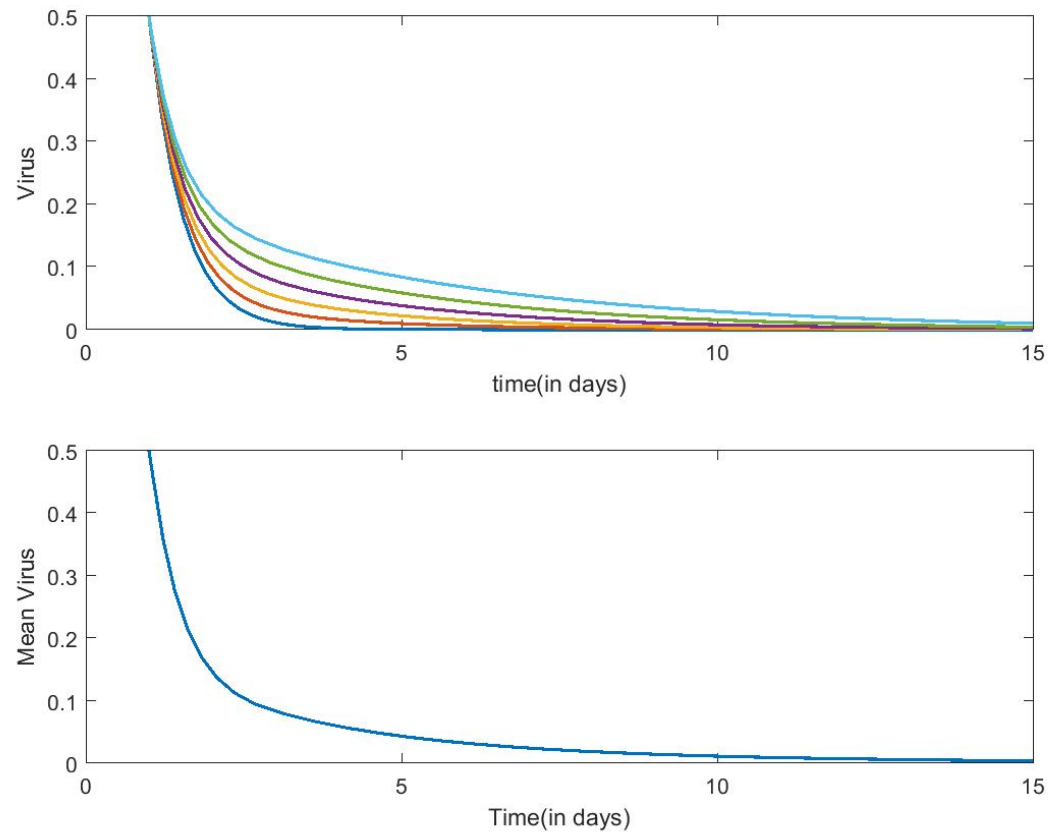

Figure 7: The virus count for values of $k$ from 0.01 to 3 and the mean value of the virus count. The rest of the parameters are chosen from table 1. 

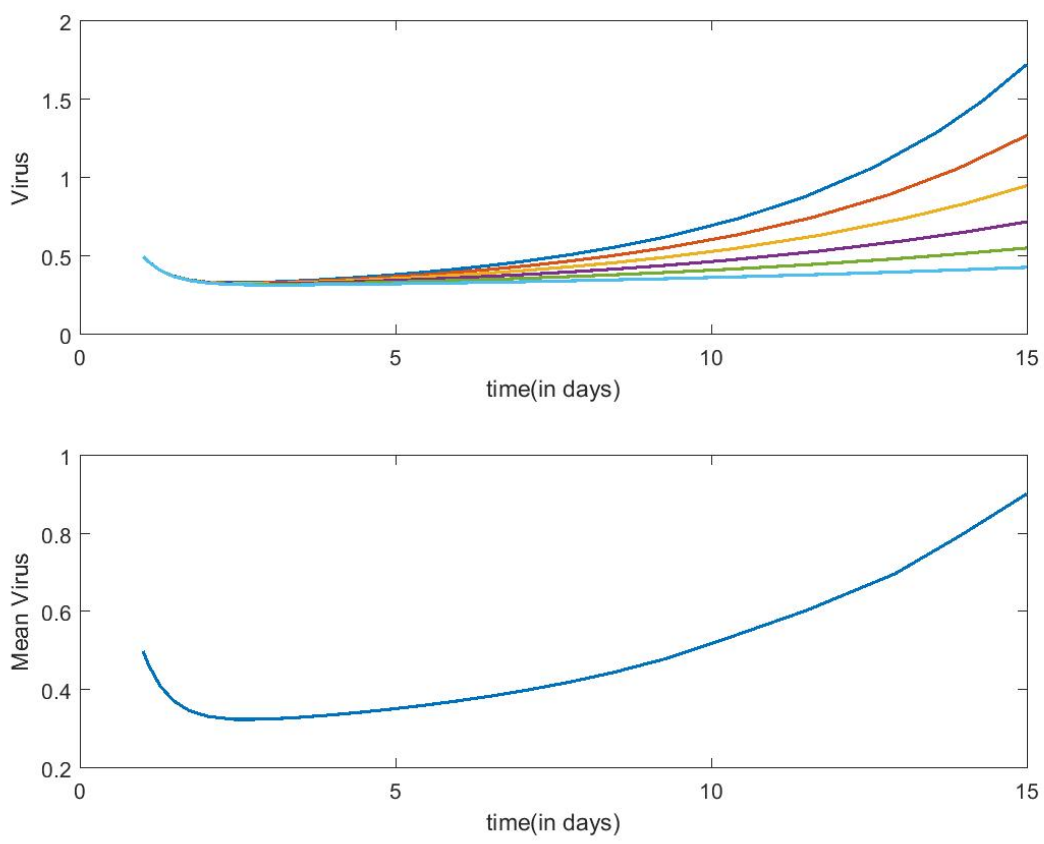

Figure 8: The virus count for values of $q$ from 0.001 to 0.28 and the mean value of the virus count. The rest of the parameters are chosen from table 1.
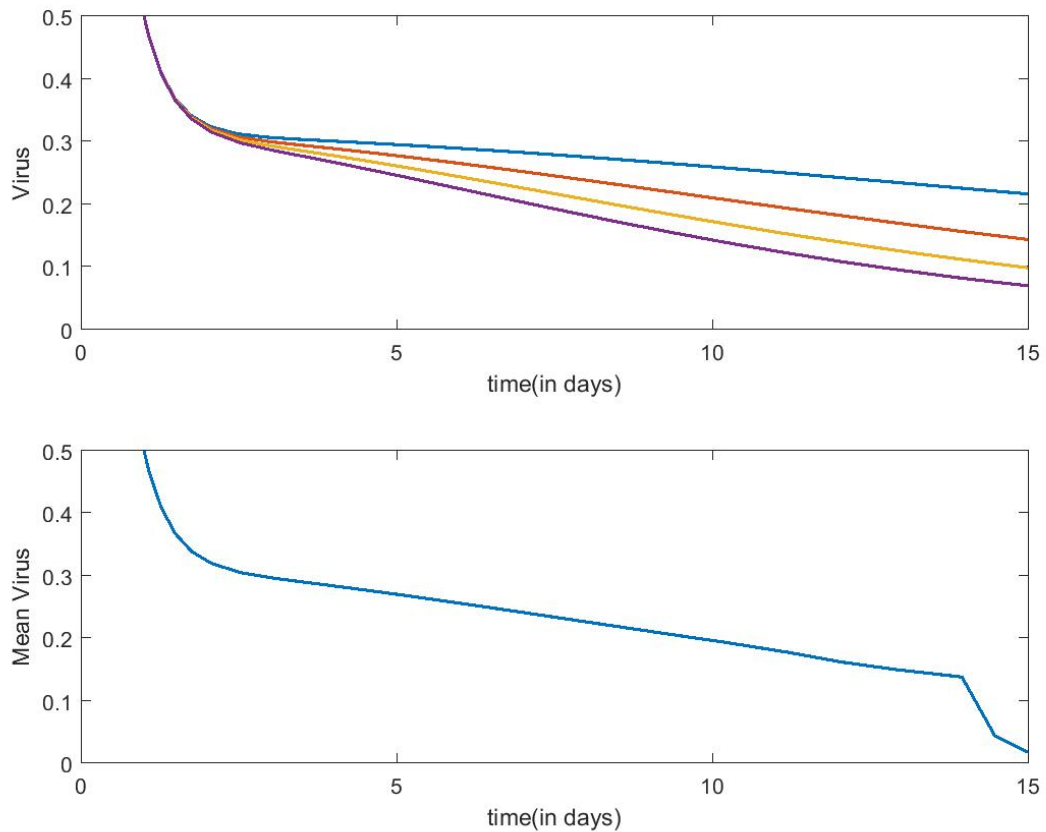

Figure 9: The virus count for values of $q$ from 0.4 to 0.7 and the mean value of the virus count. The rest of the parameters are chosen from table 1.

\section{Conclusions}

In this study, a conceptual model was developed using a system of non-linear ordinary differential equations to understand the dynamics of dengue virus. The ordinary differential equations describe the dynamical 
behaviour of healthy cells, infected cells, virus count, T-cells, B-cells, antibodies and interferons. The model incorporates the effects of innate and adaptive(humoral and cellular) immune responses to present the mechanism of viral infection. Also, a delay in the B-cell production has been considered.

The existence and stability conditions for the equilibrium points have been obtained. The threshold value of time delay $\left(\tau_{0}\right)$ has been computed which is critical for change in stability of the endemic state.

It has been observed that that innate response co-relates with the virus titer in the early stages of the infection which can help in an early detection of a serious disease.

Sensitivity analysis was performed to identify the crucial and sensitive parameters of the model. It was see that the burst rate of virus particles and the the rate of production of antibodies are the sensitive parameters in our system.

In the end we conclude by stating that the human immune system is very complex and the works presented in this paper could enhance our understanding of this complex immune response.

Acknowledgements: The authors dedicate this paper to the founder chancellor of SSSIHL, Bhagawan Sri Sathya Sai Baba. The corresponding author also dedicates this paper to his loving elder brother D. A. C. Prakash who still lives in his heart.

Author's statement: Conflict of interest: Authors state no conflict of interest.

\section{References}

[1] Sebastian Aguirre, Ana M Maestre, Sarah Pagni, Jenish R Patel, Timothy Savage, Delia Gutman, Kevin Maringer, Dabeiba Bernal-Rubio, Reed S Shabman, Viviana Simon, et al. Denv inhibits type i ifn production in infected cells by cleaving human sting. PLoS pathog, 8(10):e1002934, 2012.

[2] Mathieu Andraud, Niel Hens, Christiaan Marais, and Philippe Beutels. Dynamic epidemiological models for dengue transmission: a systematic review of structural approaches. PloS one, 7(11):e49085, 2012.

[3] Tal I Arnon, Hagit Achdout, Niva Lieberman, Roi Gazit, Tsufit Gonen-Gross, Gil Katz, Ahuva Bar-Ilan, Noga Bloushtain, Marianna Lev, Aviva Joseph, et al. The mechanisms controlling the recognition of tumor-and virus-infected cells by nkp46. Blood, 103(2):664-672, 2004.

[4] Philippe Barbazan, M Guiserix, W Boonyuan, W Tuntaprasart, D Pontier, and J-P Gonzalez. Modelling the effect of temperature on transmission of dengue. Medical and veterinary entomology, 24(1):66-73, 2010.

[5] Rotem Ben-Shachar and Katia Koelle. Minimal within-host dengue models highlight the specific roles of the immune response in primary and secondary dengue infections. Journal of the Royal Society Interface, 12(103):20140886, 2015.

[6] Hannah E Clapham, Vianney Tricou, Nguyen Van Vinh Chau, Cameron P Simmons, and Neil M Ferguson. Within-host viral dynamics of dengue serotype 1 infection. Journal of the Royal Society Interface, 11(96):20140094, 2014.

[7] Sharone Green, Sathit Pichyangkul, David W Vaughn, Siripen Kalayanarooj, Suchitra Nimmannitya, Ananda Nisalak, Ichiro Kurane, Alan L Rothman, and Francis A Ennis. Early cd69 expression on peripheral blood lymphocytes from children with dengue hemorrhagic fever. The Journal of infectious diseases, 180(5):1429-1435, 1999.

[8] Tanvi P Gujarati and G Ambika. Virus antibody dynamics in primary and secondary dengue infections. Journal of mathematical biology, 69(6):1773-1800, 2014.

[9] José Lourenço, Warren Tennant, Nuno R Faria, Andrew Walker, Sunetra Gupta, and Mario Recker. Challenges in dengue research: A computational perspective. Evolutionary applications, 11(4):516-533, 2018.

[10] Arti Mishra and Sunita Gakkhar. A micro-epidemic model for primary dengue infection. Communications in Nonlinear Science and Numerical Simulation, 47:426-437, 2017.

[11] Erika Navarro-Sánchez, Philippe Desprès, and Leticia Cedillo-Barrón. Innate immune responses to dengue virus. Archives of medical research, 36(5):425-435, 2005.

[12] Ryan Nikin-Beers and Stanca M Ciupe. The role of antibody in enhancing dengue virus infection. Mathematical biosciences, 263:83-92, 2015. 
[13] Nuning Nuraini, Hengki Tasman, Edy Soewono, and Kuntjoro Adji Sidarto. A with-in host dengue infection model with immune response. Mathematical and Computer Modelling, 49(5-6):1148-1155, 2009.

[14] Kasia A Pawelek, Giao T Huynh, Michelle Quinlivan, Ann Cullinane, Libin Rong, and Alan S Perelson. Modeling within-host dynamics of influenza virus infection including immune responses. PLoS Comput Biol, 8(6):e1002588, 2012.

[15] SD Perera and SSN Perera. Simulation model for dynamics of dengue with innate and humoral immune responses. Computational and mathematical methods in medicine, 2018, 2018.

[16] Andrea K Perry, CHEN Gang, Dahai Zheng, TANG Hong, and Genhong Cheng. The host type i interferon response to viral and bacterial infections. Cell research, 15(6):407-422, 2005.

[17] Caroline Petitdemange, Nadia Wauquier, Juliana Rey, Baptiste Hervier, Eric Leroy, and Vincent Vieillard. Control of acute dengue virus infection by natural killer cells. Frontiers in immunology, 5:209, 2014. 